\title{
Repercusiones producto del desconocimiento del sistema de referencia en la información geoespacial de Costa Rica: utilidad de los metadatos
}

\author{
Impacts resulting from lack of knowledge about the reference \\ system in geospatial information of Costa Rica: utility of metadata
}

\author{
Bepsy Cedeño-Montoya ${ }^{1}$ \\ Jorge Moya-Zamora ${ }^{2}$ \\ Universidad Nacional, Costa Rica
}

http://dx.doi.org/10.15359/rgac.60-1.2

\begin{abstract}
RESUMEN
El aprovechamiento máximo de la información geoespacial depende, entre otros aspectos, del conocimiento de su referencia geodésica, la cual es indispensable para poder convertirla y transformarla a otras referencias en caso de que sea necesario. En el caso particular de Costa Rica, existe una importante cantidad de información que está vinculada al datum convencional de Ocotepeque con sus respectivas proyecciones Lambert Costa Rica Norte (LCRN) y Lambert Costa Rica Sur (LCRS), y en la última década, la información nacional que se ha generado está referenciada al datum nacional oficial CR05 y su respectiva proyección cartográfica CRTM05 (Moya y Cedeño, 2016b). Las facilidades que ofrecen los Sistemas de Información Geográfica (SIG) propietarios o en versiones libres, constituyen una excelente herramienta no solo por la rapidez en los distintos procesos de la información, sino que, además, y quizás lo más importante, les brindan a los usuarios una enorme cantidad de resultados producto de sus múltiples capacidades de análisis espacial.
\end{abstract}

1 Máster, Escuela de Ciencias Geográficas. Universidad Nacional, Costa Rica. Correo electrónico: bepsy. cedeno.montoya@una.cr

2 Dr.-Ing., Centro Nacional de Procesamiento de Datos GNSS. Escuela de Topografía, Catastro y Geodesia. Universidad Nacional, Costa Rica, Costa Rica. Correo electrónico: jorge.moya.zamora@una.cr

Aprobado: 24 de julio de 2017 
Bepsy Cedeño-Montoya, Jorge Moya-Zamora. Impacts resulting from lack of knowledge about the reference system in geospatial information of Costa Rica: utility of metadata

Sin embargo, es indispensable que los SIG estén adecuadamente configurados, de manera que se pueda extraer la mayor cantidad de datos posibles con la certeza de que la información de partida está correctamente georreferenciada, o de que el proceso previo de georreferenciación es posible y correcto. Para esta tarea, es fundamental el conocimiento de los metadatos de manera de conocer el origen de la información. El objetivo principal de este trabajo es mostrarle a una gran cantidad de usuarios de los SIG, las posibles repercusiones que se tendrán por un inadecuado proceso de conversión y transformación de coordenadas.

Palabras clave: SIG, metadatos, sistema de referencia en Costa Rica, proyección, datum

\begin{abstract}
The maximum use of geospatial information depends on knowing the geodetic reference, which is essential to transform it into other references -if needed. In the specific case of Costa Rica, there is a significant amount of information that is linked to conventional datum Ocotepeque with their corresponding projections Lambert Costa Rica Norte (LCRN) and Lambert Costa Rica Sur (LCRS). And, in the last decade, the national information that has been generated is referenced to the official national datum CR05 and its corresponding map projection CRTM05 (Moya y Cedeño, 2016). The facilities offered by the Geographic Information Systems (GIS) -proprietary or free versions, are an excellent tool not only due to the speed in the different information processes, but also, and perhaps most importantly, because they give users a huge amount of results due to their multiple spatial analysis capabilities. However, it is essential that GIS are properly configured so that it is possible to extract as much information as possible and knowing that the initial information is properly georeferenced or that the prior georeference process is possible and correct. For this task, knowing the metadata is essential in order to know the origin of the information. The main purpose of this work is to show a lot of GIS users, the possible impacts resulting from the incorrect conversion process and coordinate transformation.
\end{abstract}

Keywords: SIG; metadata; reference systems in Costa Rica, projection, datum

\title{
Introducción
}

El uso de los Sistemas de Información Geográfica (SIG) para el procesamiento y análisis de datos geoespaciales se ha convertido prácticamente en la norma a nivel mundial. La facilidad que ofrecen los diferentes sistemas SIG para la visualización y posterior tratamiento de la información, ha generado que cada vez más usuarios empleen estas herramientas en sus proyectos particulares. Sin embargo, es lógico pensar que la información geoespacial debe estar adecuadamente referida para poder, si es el caso, transformarla de un sistema a otro sin incurrir en errores, que, dependiendo de la finalidad, pueden ser considerables. Se presentan a continuación los resultados de una serie de conversiones y posteriores transformaciones usando el software ArcGIS, versión 10.3 de la casa comercial ESRI y licencia de la Universidad Nacional de Costa Rica, aplicadas sobre un conjunto de datos geodésicos oficiales de Costa Rica y las 
discrepancias que se obtuvieron al considerar diferentes variantes de una común aplicación por parte de un gran sector de los usuarios. El objetivo principal de este estudio es reafirmar la importancia que tienen los metadatos para poder hacer un adecuado proceso de transformación, sobre todo en un país como Costa Rica, donde se ha generado información referida a muchos datum y proyecciones cartográficas (Dörries y Roldán, 2004) y (Moya y Cedeño, 2016b).

\section{Metodología \\ 1. Información de partida}

Se consideraron dos conjuntos de datos oficiales, conformados principalmente por coordenadas geodésicas: el primero, referido al antiguo datum Ocotepeque, elipsoide de Clarke 1866 y conformado por las cabeceras de los distritos de Costa Rica (Costa Rica, 2001) y las esquinas noroeste de las hojas del mapa básico escala 1:50000. El segundo grupo, referido al actual datum nacional CR05, elipsoide WGS84 consistió de los vértices de la red geodésica de primer y segundo orden de Costa Rica.

Adicionalmente, se tomaron las respectivas coordenadas cartográficas de estos datos en las proyecciones Lambert Costa Rica Norte (LCRN) y en Costa Rica Transversa Mercator (CRTM05) respectivamente. En el cuadro 1, se resumen el tipo y cantidad de datos utilizados en las distintas variantes de transformación y en las figuras 1 y 2 se presentan la distribución espacial de cada grupo.

Cuadro 1. Tipo y fuente de los datos utilizados

\begin{tabular}{|l|l|l|l|l|}
\hline \multicolumn{1}{|c|}{ Fuente } & \multicolumn{1}{|c|}{ Datum } & \multicolumn{1}{|c|}{ Elipsoide } & Proyección & Cantidad \\
\hline $\begin{array}{l}\text { Coordenadas geodésicas }(\varphi, \lambda) \text { y } \\
\text { cartográficas }(\mathrm{N}, \text { E) de las ciudades cabecera } \\
\text { de los distritos de Costa Rica }\end{array}$ & Ocotepeque & Clarke 1866 & $\begin{array}{l}\text { LCRN y } \\
\text { LCRS }\end{array}$ & 426 \\
\hline $\begin{array}{l}\text { Coordenadas geodésicas }(\varphi, \lambda) \text { y } \\
\text { cartográficas (N, E) de las esquinas noroeste } \\
\text { de las hojas del mapa básico de Costa Rica, } \\
\text { escala 1:50000 }\end{array}$ & Ocotepeque & Clarke 1866 & $\begin{array}{l}\text { LCRN y } \\
\text { LCRS }\end{array}$ & 128 \\
\hline $\begin{array}{l}\text { Coordenadas geodésicas }(\varphi, \lambda) \text { y } \\
\text { cartográficas }(\mathrm{N}, \text { E) de los puntos de la red de } \\
\text { primer orden y segundo orden de Costa Rica }\end{array}$ & CR05 & WGS84 & CRTM05 & 86 \\
\hline
\end{tabular}

Fuente: Elaboración propia 
Bepsy Cedeño-Montoya, Jorge Moya-Zamora. Impacts resulting from lack of knowledge about the reference system in geospatial information of Costa Rica: utility of metadata

Figura 1. Ubicación de las cabeceras de los distritos y de las esquinas de las hojas del mapa básico de Costa Rica

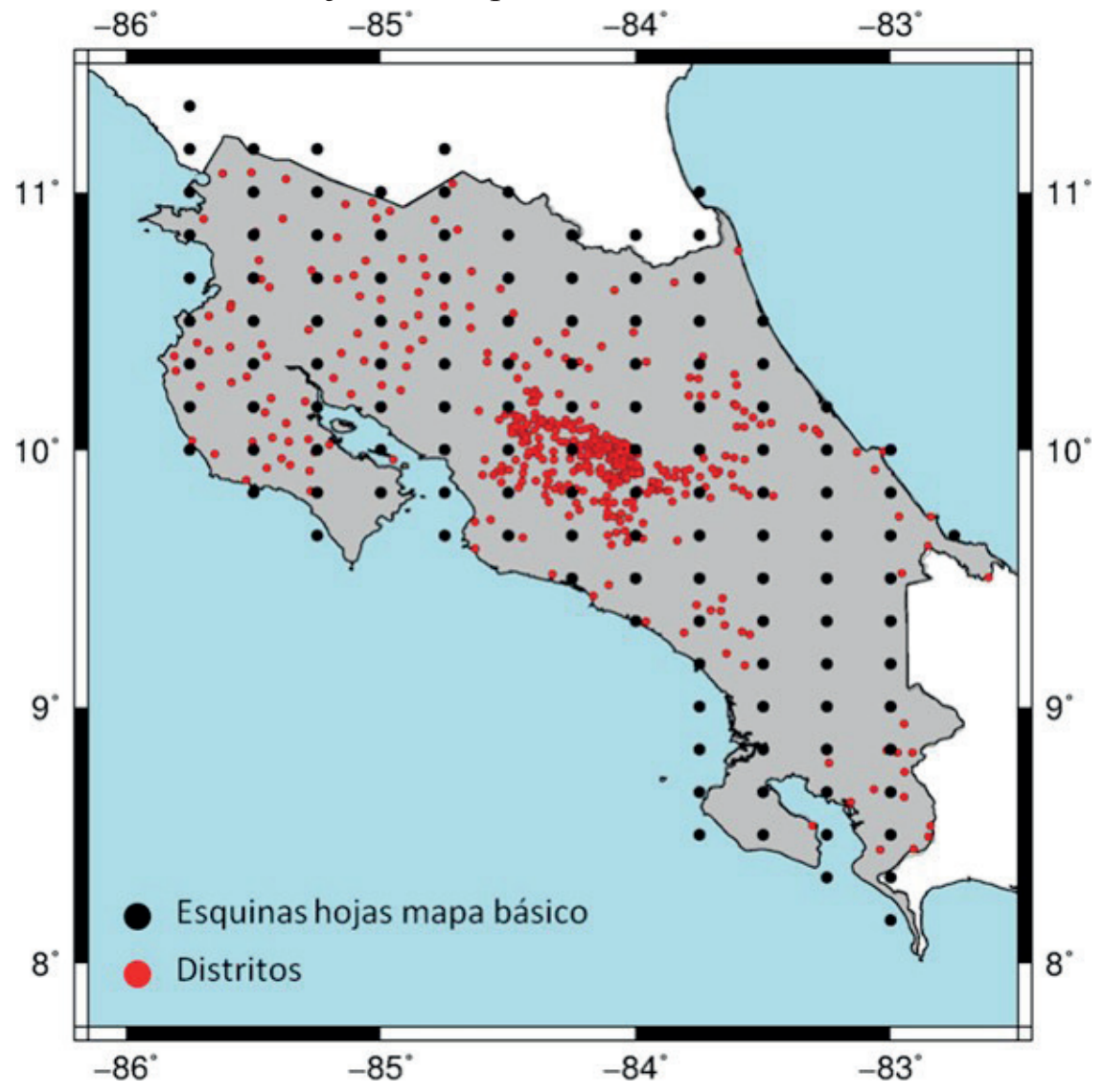

Fuente: Elaboración propia 
Figura 2. Ubicación de los puntos de la red geodésica de primer y segundo orden de Costa Rica

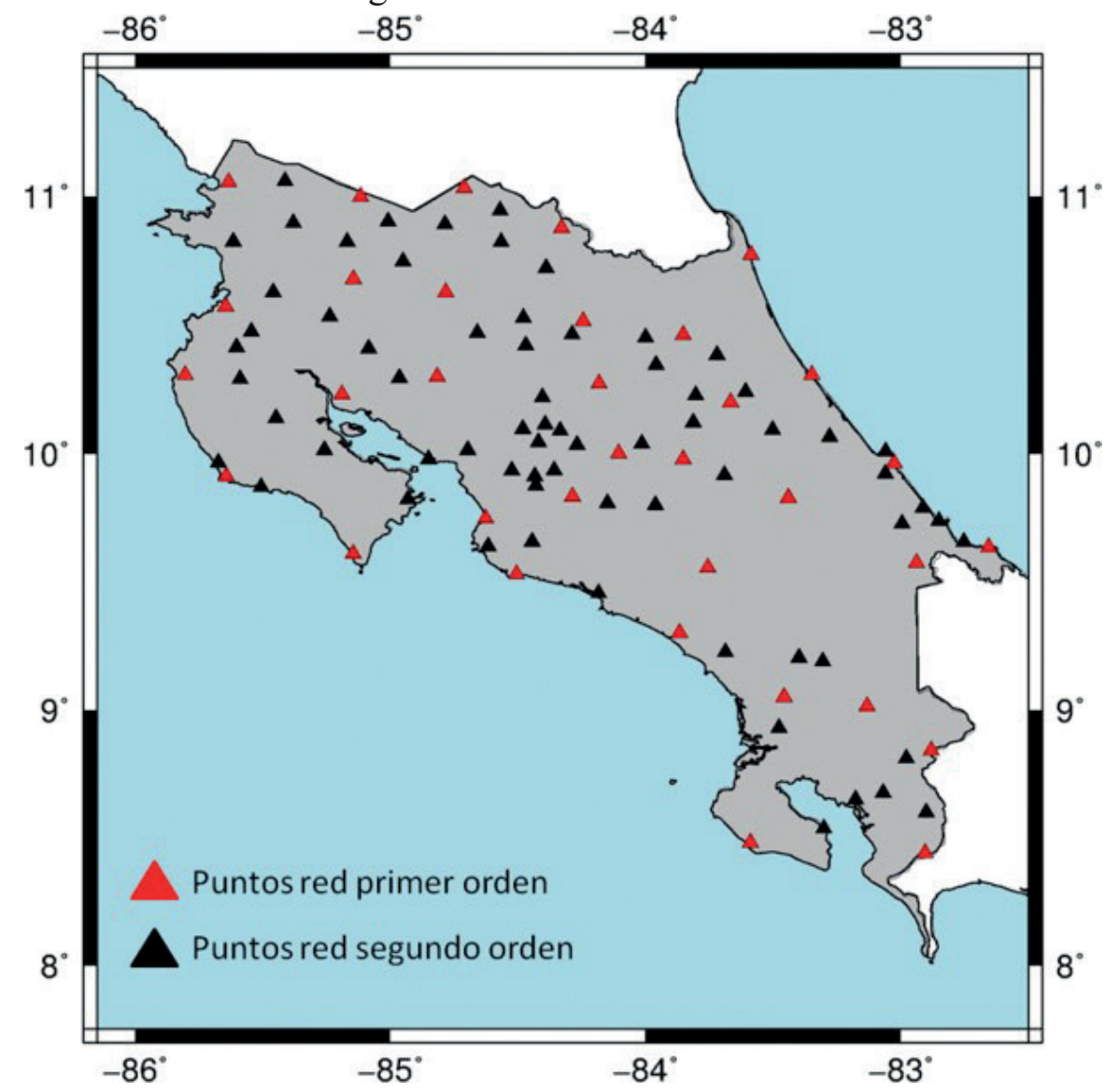

Fuente: Elaboración propia

\section{Procesos de conersión y transformación}

Los diferentes procesos efectuados se denominaron internamente como: caso A, caso B, caso $\mathrm{C}$ y caso $\mathrm{D}$, en los que se realizaron diferentes conversiones y transformaciones entre sistemas de coordenadas y planos utilizando el programa ArcGIS 10.3. Estos procesos se efectuaron aplicando herramientas de Projections and Transformations del Data Management Tools contenido en el ArcToolBox de ArcGIS. 
Bepsy Cedeño-Montoya, Jorge Moya-Zamora. Impacts resulting from lack of knowledge about the reference system in geospatial information of Costa Rica: utility of metadata

A. Coordenadas geodésicas $(\varphi, \lambda)$ de las ciudades cabecera de los distritos de Costa Rica y de las esquinas noroeste de las hojas del mapa básico de Costa Rica, escala 1:50000 referidas al datum Ocotepeque, elipsoide Clarke 1866.

1. Se partió de un archivo en formato Excel en el que se tienen las coordenadas geodésicas de esta serie de objetos, indicados en grados decimales. Comúnmente las coordenadas expresadas en estas unidades son asumidas, por una gran mayoría de usuarios SIG, como coordenadas geográficas correspondientes al elipsoide WGS84. Para mostrar las diferencias producto de este supuesto erróneo, se consideraron inicialmente las coordenadas geodésicas como referidas a WGS84. El proceso que se siguió fue el siguiente:

1.1. Se agregó al ArcMap la tabla externa y con la herramienta Display XY Data se generó un tema que muestra, en forma de puntos, la ubicación espacial de los datos.

1.2. Utilizando la opción Define Projection de las herramientas de proyecciones se definió el tema como WGS84. Debe aclararse que WGS84 es un elipsoide y no datum.

1.3. Posteriormente, se transformaron los datos definidos como WGS84 hacia el sistema CRTM05, aplicando la herramienta Project y la variable de transformación CR05_to_WGS84_1 que el programa coloca por defecto. Se agregaron a la tabla de atributos del tema los valores de coordenadas cartográficas de cada punto. En el cuadro 2, se presentan los valores extremos y el promedio, así como la resultante y su error.

Cuadro 2. Valores extremos de las diferencias en coordenadas cartográficas CRTM05, considerando como superficie de partida WGS84 en lugar de Clarke 1866

\begin{tabular}{|c|c|c|c|c|c|c|}
\hline \multicolumn{7}{|c|}{$\begin{array}{l}\text { Coordenadas de partida: geodésicas }(\varphi, \lambda) \\
\text { Datum: Ocotepeque, Elipsoide: Clarke } 1866\end{array}$} \\
\hline $\begin{array}{l}\text { Coordenada } \\
\text { CRTM05 }\end{array}$ & $\begin{array}{l}\text { Mínimo } \\
\text { [mm] }\end{array}$ & $\begin{array}{l}\text { Máximo } \\
{[\mathrm{mm}]}\end{array}$ & $\begin{array}{l}\text { Promedio } \\
\text { [mm] }\end{array}$ & $\begin{array}{l}\text { Error } \\
{[\mathrm{mm}]}\end{array}$ & $\begin{array}{l}\text { Resultante } \\
{[\mathrm{mm}]}\end{array}$ & $\begin{array}{l}\text { Error } \\
{[\mathrm{mm}]}\end{array}$ \\
\hline Norte & 127784,2 & 167067,4 & 157047,6 & 242,4 & \multirow{2}{*}{265028,2} & \multirow{2}{*}{225,0} \\
\hline Este & $-262196,3$ & $-206722,1$ & $-213485,4$ & 215,0 & & \\
\hline
\end{tabular}

Fuente: Elaboración propia 

referencia en la información geoespacial de Costa Rica: utilidad de los metadatos

En la figura 3 se muestra la dirección del vector diferencia entre ambos conjuntos de coordenadas. Se aprecia predominantemente que las discrepancias van en la dirección sureste con un promedio de $265 \mathrm{~m}$.

Figura 3. Comportamiento de los vectores diferencia para coordenadas cartográficas CRTM05 asignando como superficie de referencia el

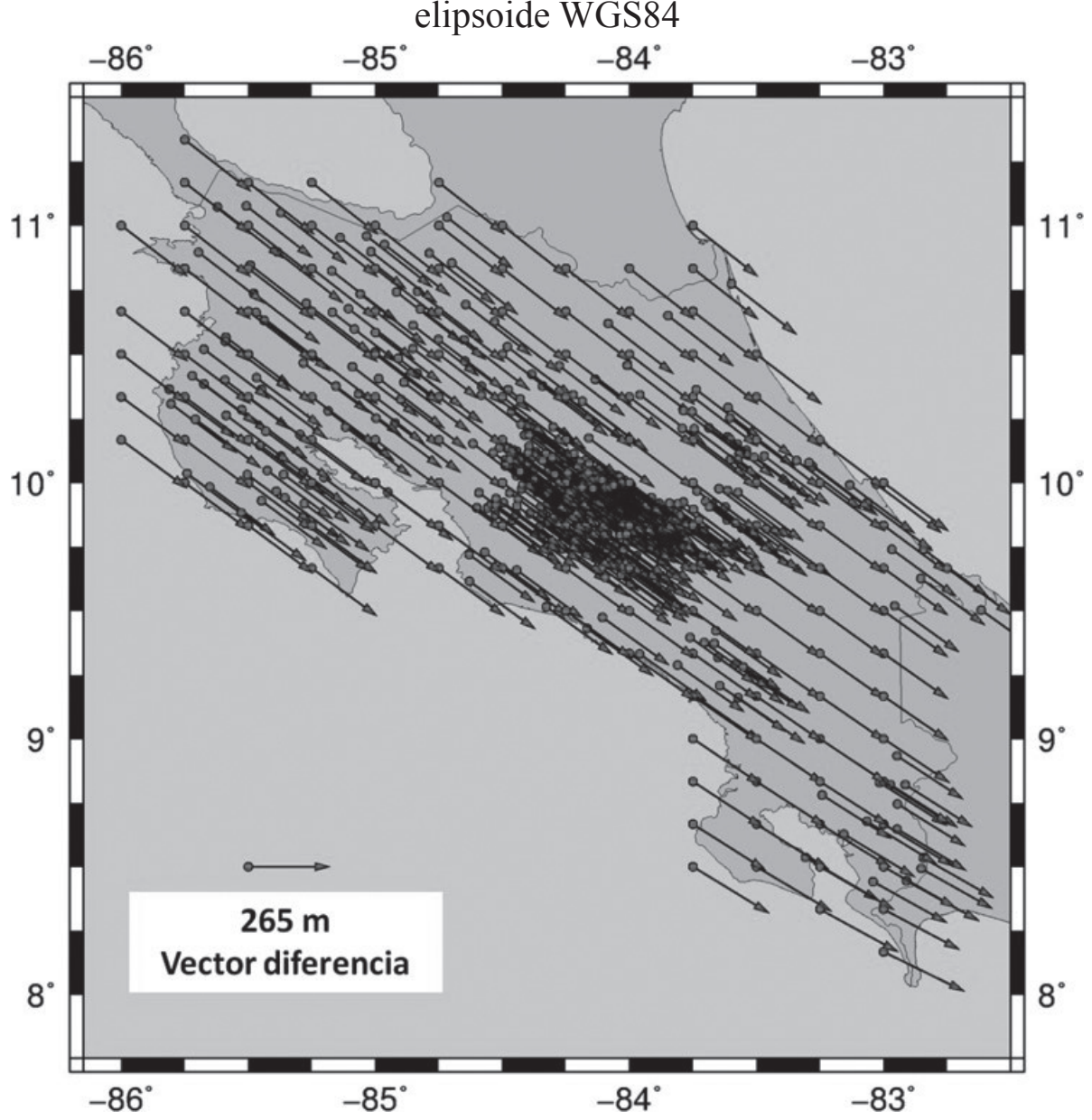

Fuente: Elaboración propia

2. Al archivo en CRTM05 se le realizó nuevamente otra transformación de los puntos hacia el sistema LCRN, seleccionando la variable de transformación CR05_to_Ocotepeque_1935_MB, y se calcularon las 
Bepsy Cedeño-Montoya, Jorge Moya-Zamora. Impacts resulting from lack of knowledge about the reference system in geospatial information of Costa Rica: utility of metadata

coordenadas cartográficas para cada registro de la tabla de atributos. En el cuadro 3, se presentan los valores extremos y el promedio, así como la resultante al comparar con las coordenadas cartográficas oficiales.

Cuadro 3. Valores extremos de las diferencias en coordenadas cartográficas LCRN, considerando como superficie de partida WGS84 en lugar de Clarke 1866, usando la variable CR05_to_Ocotepeque_1935_MB

\begin{tabular}{|c|c|c|c|c|c|c|}
\hline \multicolumn{7}{|c|}{$\begin{array}{c}\text { Coordenadas de partida: geodésicas }(\varphi, \lambda) \\
\text { Datum: Ocotepeque } \\
\text { Elipsoide: Clarke 1866 }\end{array}$} \\
\cline { 1 - 5 } $\begin{array}{c}\text { Coordenada } \\
\text { LCRN }\end{array}$ & $\begin{array}{c}\text { Mínimo } \\
{[\mathbf{m m}]}\end{array}$ & $\begin{array}{c}\text { Máximo } \\
{[\mathbf{m m}]}\end{array}$ & $\begin{array}{c}\text { Promedio } \\
{[\mathbf{m m}]}\end{array}$ & $\begin{array}{c}\text { Error } \\
{[\mathbf{m m}]}\end{array}$ & $\begin{array}{c}\text { Resultante } \\
{[\mathbf{m m}]}\end{array}$ & $\begin{array}{c}\text { Error } \\
{[\mathbf{m m}]}\end{array}$ \\
\cline { 1 - 5 } Norte & 140494,7 & 169031,8 & 156886,3 & 191,3 & 264536,3 & 113,9 \\
\hline Este & $-214049,7$ & $-212078,9$ & $-212993,2$ & 12,8 & \\
\hline
\end{tabular}

Los resultados de los cuadros 2 y 3 evidencian diferencias promedio de 265 m, confirmando los resultados dados por Dörries y Roldán (2004), como valor promedio entre el datum convencional de Ocotepeque y el datum CR98. El proceso indicado anteriormente, arrastra un error desde el inicio, ya que es incorrecto asumir que las coordenadas geodésicas corresponden al elipsoide WGS84. Se debe revisar cuidadosamente la fuente de los datos, ya que las ciudades cabecera de los distritos de Costa Rica, así como las esquinas noroeste de las hojas del mapa básico de Costa Rica, están referidas al datum Ocotepeque, elipsoide Clarke 1866.

Por ejemplo, se puede tener coordenadas cartográficas referidas al datum Ocotepeque y elipsoide Clarke 1866, pero proyectadas a otro plano cartográfico diferente al plano conforme Lambert. Es decir, directamente en el SIG, un usuario podría sin mayor complicación generar un sistema de coordenadas planas basado en una proyección Lambert, pero usando como datum CR05 y elipsoide WGS84. Lo anterior no implica que los datos deban manipularse antojadizamente, ya que, si se está trabajando con información geoespacial, deben respetarse los parámetros de cada una de las proyecciones nacionales (Moya y Cedeño, 2016).

3. Considerando lo anterior, se realizó nuevamente el ejercicio de transformación de coordenadas de manera correcta, el cual contempló los siguientes pasos: 
Bepsy Cedeño-Montoya, Jorge Moya-Zamora. Repercusiones producto del desconocimiento del sistema de referencia en la información geoespacial de Costa Rica: utilidad de los metadatos

3.1. Se definió la vista de ArcMap (en el menú View: Data Frame Properties: Coordinate Systems) seleccionando a Ocotepeque 1935 como sistema de referencia, tal como se muestra en la figura 4.

Figura 4. Captura de pantalla de la opción Data Frame Properties, menú View de ArcMap

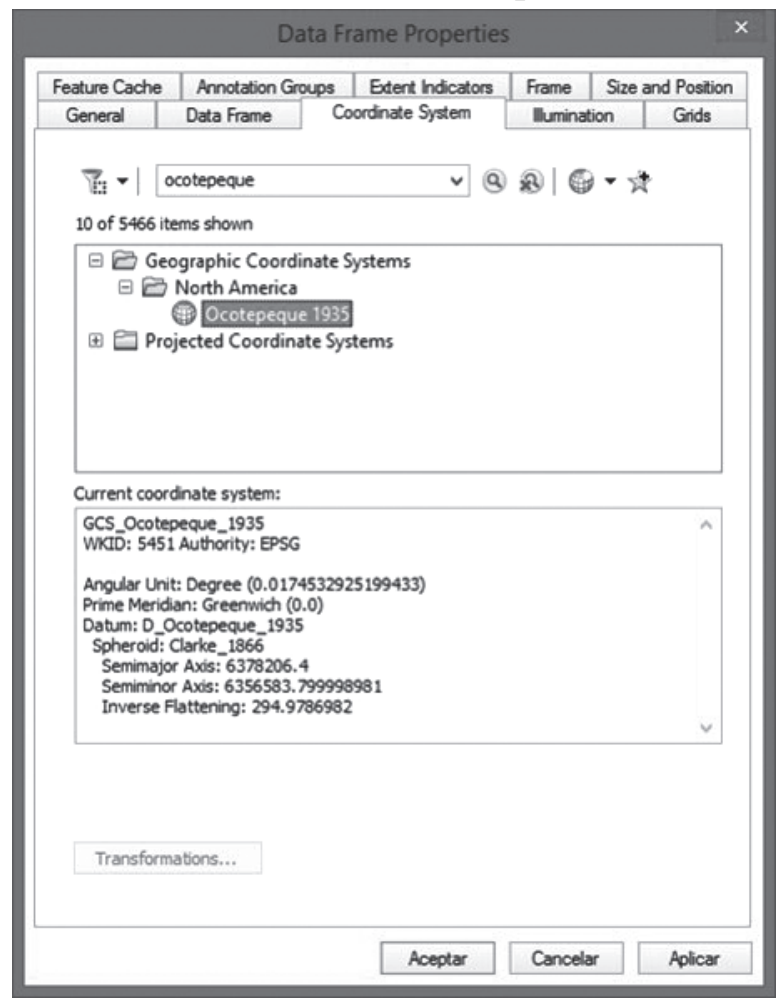

Fuente: Elaboración propia

3.2. Se añadió la tabla externa a ArcMap y con la herramienta Display XY Data se generó el tema que muestra la posición geográfica de los datos. Al estar definida la vista los datos asumen automáticamente el mismo sistema, es decir, Ocotepeque 1935.

3.3. Se realizó la transformación de coordenadas geodésicas a coordenada planas en LCRN, utilizando la herramienta Project, y como ambos sistemas tienen en común el datum Ocotepeque 1935 el programa no solicita variable de transformación 
Bepsy Cedeño-Montoya, Jorge Moya-Zamora. Impacts resulting from lack of knowledge about the reference system in geospatial information of Costa Rica: utility of metadata

alguna. Se agregaron a la tabla de atributos del tema los valores de coordenadas cartográficas de cada punto.

En el cuadro 4 se muestran los resultados de este proceso al compararlos con las coordenadas cartográficas oficiales. Tomando adecuadamente el datum y la superficie de origen de los datos en el proceso dentro del SIG, se aprecia como los valores promedio de las diferencias en las coordenadas norte y este, así como la resultante son prácticamente nulos, como es de esperarse.

Cuadro 4. Valores extremos de las diferencias en coordenadas cartográficas LCRN, considerando como superficie de partida WGS84 en lugar de Clarke 1866

\begin{tabular}{|c|c|c|c|c|c|c|}
\hline \multicolumn{7}{|c|}{$\begin{array}{c}\text { Coordenadas de partida: geodésicas }(\varphi, \lambda) \\
\text { Datum: Ocotepeque } \\
\text { Elipsoide: Clarke 1866 }\end{array}$} \\
\cline { 1 - 4 } $\begin{array}{c}\text { Coordenada } \\
\text { LCRN }\end{array}$ & $\begin{array}{c}\text { Mínimo } \\
{[\mathbf{m m}]}\end{array}$ & $\begin{array}{c}\text { Máximo } \\
{[\mathbf{m m}]}\end{array}$ & $\begin{array}{c}\text { Promedio } \\
{[\mathbf{m m}]}\end{array}$ & $\begin{array}{c}\text { Error } \\
{[\mathbf{m m}]}\end{array}$ & $\begin{array}{c}\text { Resultante } \\
{[\mathbf{m m}]}\end{array}$ & $\begin{array}{c}\text { Error } \\
{[\mathbf{m m}]}\end{array}$ \\
\hline Norte & $-48,2$ & 7,1 & $-0,7$ & 0,2 & 1,5 & 0,1 \\
\hline Este & $-3,9$ & 16,2 & 1,3 & 0,1 & & \\
\hline
\end{tabular}

Fuente: Elaboración propia

\section{B. Coordenadas geodésicas $(\varphi, \lambda)$ y cartográficas $(\mathbf{N}, \mathbf{E})$ de los puntos de la red de primer orden y segundo orden de Costa Rica, datum CR05, elipsoide WGS84.}

1. Los datos de partida para este segundo caso son las coordenadas geodésicas oficiales de los puntos de la red de primer y segundo orden de Costa Rica, indicadas en grados decimales, y como se mencionó en el inicio del caso A, comúnmente las coordenadas así expresadas son asumidas por parte de muchos usuarios de SIG, como coordenadas geodésicas correspondientes al elipsoide WGS84. Los pasos realizados fueron los siguientes: 
1.1. Se agregó al ArcMap la tabla externa y con la herramienta Display XY Data se generó un tema que muestra, en forma de puntos, la ubicación espacial de los datos.

1.2. Utilizando la opción Define Projection de las herramientas de proyecciones se definió el tema como WGS84.

1.3. Luego se transformaron los datos con coordenadas geográficas definidas como WGS84 hacia el sistema CRTM05, aplicando la herramienta Project y la variable de transformación CR05 to_WGS84_1. Se agregaron a la tabla de atributos del tema los valores de coordenadas cartográficas de cada punto. Como es de esperarse, las diferencias luego de comparar las coordenadas cartográficas de este proceso con los valores oficiales son nulas, ya que se está partiendo de un conjunto de coordenadas geodésicas vinculadas al datum CR05, elipsoide WGS84.

1.4. Al archivo en CRTM05 resultante, se le realizó otra transformación hacia el sistema LCRN, con la variable de transformación CR05_to_Ocotepeque_1935_MB, y se calcularon las coordenadas cartográficas para cada registro. Como producto de la comparación con los valores oficiales se obtuvieron diferencias para la coordenada norte que van desde los $-1,3 \mathrm{~m}$ a los $2,7 \mathrm{~m}$ y para la coordenada este entre los $-4,7 \mathrm{~m}$ y $1,1 \mathrm{~m}$. Para esta comparación se excluyeron los puntos que se localizan en la zona definida para la cartográfica LCRS. En la figura 5 se representan estas diferencias por medio de los correspondientes vectores diferencia. 
Bepsy Cedeño-Montoya, Jorge Moya-Zamora. Impacts resulting from lack of knowledge about the reference system in geospatial information of Costa Rica: utility of metadata

Figura 5. Comportamiento de los vectores diferencias para coordenadas cartográficas LCRN para algunos de los puntos de la red geodésica de primer orden y segundo, usando la variable CR05_to_Ocotepeque_1935_MB

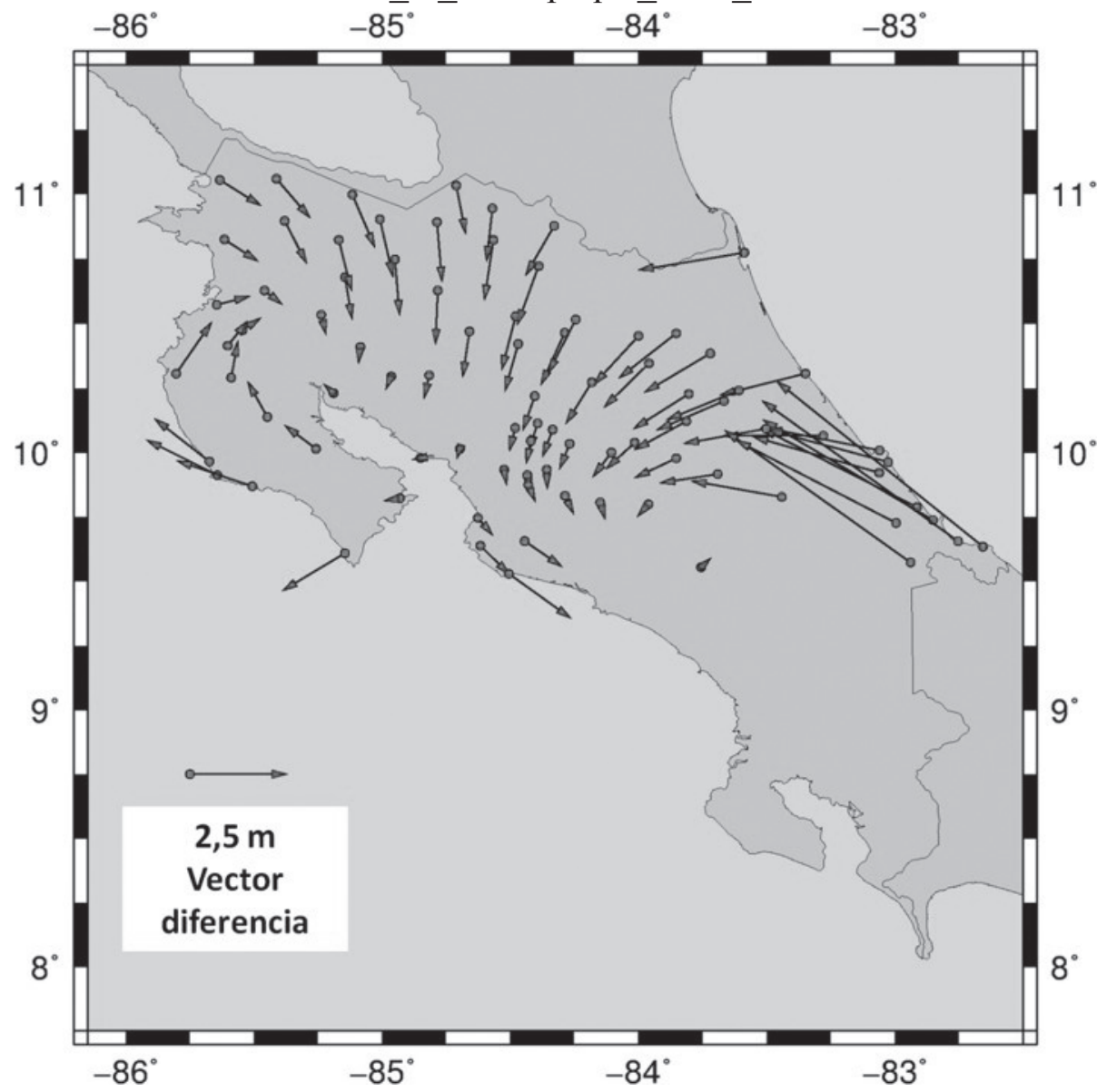

Fuente: Elaboración propia

C. Coordenadas cartográficas $(\mathrm{N}, \mathrm{E})$ de los puntos de la red de primer orden y segundo orden de Costa Rica, datum CR05, elipsoide WGS84.

1. Como en los casos anteriores, se parte de un archivo en formato de Excel con las coordenadas en sistema CRTM05 de los puntos de la 
red de primer orden y segundo orden del país. A este archivo se le aplicaron los siguientes pasos:

1.1. Agregar la tabla a ArcMap, y a las coordenadas que se despliegan como puntos se les define el sistema de proyección cartográfica CRTM05.

1.2. Se reproyectaron los datos de CRTM05 a LCRN con la variable CR05_to_Ocotepeque_1935_MB.

A los mismos datos se les aplicó el mismo proceso, pero utilizando la variable de transformación inversa, es decir, la Ocotepeque_1935_to_ CR05. Para ambos archivos resultantes se agregaron a la tabla de atributos los valores de coordenadas cartográficas de cada punto. Los resultados de estos procesos se presentan en el cuadro 5, donde se han tomado los valores máximos, mínimos y promedio de las diferencias usando la variable de transformación en sentido directo para las filas 3 y 4 , y en sentido inverso en las filas 6 y 7 .

Cuadro 5. Valores extremos de las diferencias en coordenadas cartográficas LCRN, considerando como superficie de partida WGS84 en lugar de Clarke 1866

\begin{tabular}{|c|c|c|c|c|c|c|}
\hline \multicolumn{7}{|c|}{$\begin{array}{c}\text { Coordenadas de partida: geodésicas }(\varphi, \lambda) \\
\text { Datum: CR05 } \\
\text { Elipsoide: WGS84 }\end{array}$} \\
\hline $\begin{array}{c}\text { Coordenada } \\
\text { LCRN }\end{array}$ & $\begin{array}{c}\text { Mínimo } \\
\text { [mm] }\end{array}$ & $\begin{array}{c}\text { Máximo } \\
\text { [mm] }\end{array}$ & $\begin{array}{l}\text { Promedio } \\
\text { [mm] }\end{array}$ & $\begin{array}{l}\text { Error } \\
{[\mathrm{mm}]}\end{array}$ & $\begin{array}{c}\text { Resultante } \\
{[\mathrm{mm}]}\end{array}$ & $\begin{array}{l}\text { Error } \\
{[\mathrm{mm}]}\end{array}$ \\
\hline Norte & $-1318,4$ & 2791,9 & $-99,2$ & 113,9 & \multirow{2}{*}{696,8} & \multirow{2}{*}{140,1} \\
\hline Este & $-4725,7$ & 1134,4 & $-689,7$ & 140,6 & & \\
\hline $\begin{array}{c}\text { Coordenada } \\
\text { LCRN } \\
\end{array}$ & $\begin{array}{c}\text { Mínimo } \\
\text { [mm] }\end{array}$ & $\begin{array}{c}\text { Máximo } \\
{[\mathrm{mm}]}\end{array}$ & $\begin{array}{c}\text { Promedio } \\
{[\mathrm{mm}]}\end{array}$ & $\begin{array}{l}\text { Error } \\
{[\mathrm{mm}]}\end{array}$ & $\begin{array}{c}\text { Resultante } \\
{[\mathrm{mm}]}\end{array}$ & $\begin{array}{l}\text { Error } \\
\text { [mm] }\end{array}$ \\
\hline Norte & $-1547,6$ & 4264,8 & $-275,4$ & 125,8 & \multirow{2}{*}{823,2} & \multirow{2}{*}{155,0} \\
\hline Este & $-5302,0$ & 1601,0 & $-775,7$ & 158,3 & & \\
\hline
\end{tabular}

Fuente: Elaboración propia 
Bepsy Cedeño-Montoya, Jorge Moya-Zamora. Impacts resulting from lack of knowledge about the reference system in geospatial information of Costa Rica: utility of metadata

D. Coordenadas cartográficas $(\mathrm{N}, \mathrm{E})$ de los puntos de la red de primer orden y segundo orden de Costa Rica, datum CR05, elipsoide WGS84.

1. Partiendo de coordenadas cartográficas oficiales en LCRN de los puntos de la red de primer orden y segundo orden del país, se aplicaron los siguientes pasos para obtener coordenadas CRTM05:

1.1. Agregar la tabla a ArcMap, las coordenadas se despliegan como puntos y se le define el sistema LCRN.

1.2. Se reproyectaron los datos de LCRN a CRTM05, utilizando la variable Ocotepeque_1935_to_CR05.

Los resultados que se obtuvieron de este proceso se compararon de varias formas. En primer lugar, la comparación directa entre las coordenadas LCRN oficiales y las obtenidas del SIG presentaron diferencias entre $-16,0 \mathrm{~m}$ y $5,0 \mathrm{~m}$ para la coordenada norte y entre $-49,0 \mathrm{~m}$ y $6,5 \mathrm{~m}$ para la coordenada este. En segundo lugar, las coordenadas obtenidas del proceso SIG se compararon con coordenadas oficiales en CRTM98, donde se obtuvo como resultado diferencias entre $-3,5 \mathrm{~m}$ y los $1,5 \mathrm{~m}$ para la coordenada norte y entre $-1,4 \mathrm{~m}$ y 5,0 $\mathrm{m}$ en la coordenada este. Finalmente, la comparación de las coordenadas oficiales con las obtenidas del SIG aplicando la variable de transformación en sentido inverso dio como resultado diferencias entre $-14,0 \mathrm{~m}$ y $6,0 \mathrm{~m}$ en la coordenada norte y entre $-48,5 \mathrm{~m}$ y $6,0 \mathrm{~m}$ para la coordenada este. En la figura 6 se muestran los vectores diferencia producto de los resultados al aplicar la variable de transformación en sentido directo e inverso. 
Bepsy Cedeño-Montoya, Jorge Moya-Zamora. Repercusiones producto del desconocimiento del sistema de referencia en la información geoespacial de Costa Rica: utilidad de los metadatos

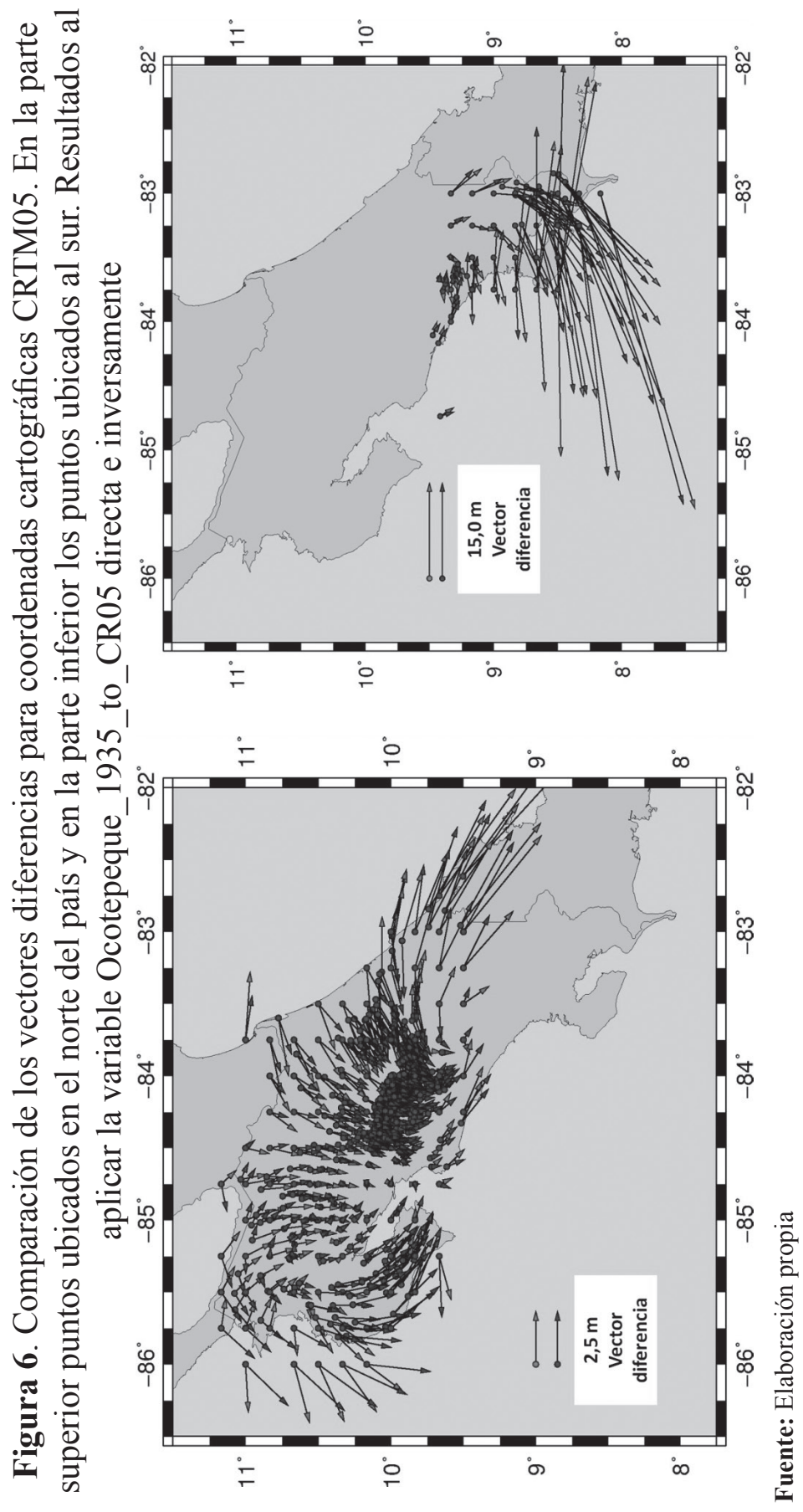


Bepsy Cedeño-Montoya, Jorge Moya-Zamora. Impacts resulting from lack of knowledge about the reference system in geospatial information of Costa Rica: utility of metadata

\section{Metadatos: su importancia para el trabajo con proyecciones cartográficas: ¿Con qué se cuenta y a dónde se puede ir?}

El Instituto Geográfico Nacional (IGN) se encuentra impulsando el desarrollo e implementación de una Infraestructura de Datos Espaciales (IDE) para Costa Rica, trabajo que oficialmente da inicio con el decreto ejecutivo 33773 JP-H-MINAE-MICIT del 7 de mayo del 2013, publicado en La Gaceta Nº134 del 12 de julio de 2013, con el que se crea el Sistema Nacional de Información Territorial (SNIT) y se busca el establecimiento y consolidación de esa IDE.

El 9 de marzo de 2016, el mismo IGN anuncia, en la página del SNIT (www.snit.go.cr), la oficialización de una serie de Normas Técnicas de Información Geográfica (NTIG) para el país. Se trata de seis documentos titulados:

- NTIG_CR01_01.2016: Sistema de Referencia Geodésico de Costa Rica

- NTIG_CR02_01.2016: Catálogo de Objetos Geográficos para Datos Fundamentales de Costa Rica

- NTIG_CR03_01.2016: Modelo de Datos Geográficos de Costa Rica, escalas $1: 1000,1: 5000$ y $1: 25000$

- NTIG_CR04_01.2016: Perfil Oficial de Metadatos Geográficos de Costa Rica.

- NTIG_CR05_01.2016: Estándares para la Publicación Web de la Información Geográfica de Costa Rica

- NTIG_CR06_01.2016: Especificaciones cartográficas para el Mapa Topográfico escala 1:25000 de Costa Rica.

Como indica el IGN (2016) en el anuncio de oficialización publicado en el SNIT, todos estos documentos son:

[...] la primera versión de una iniciativa que facilitará y adecuará el intercambio de información en el ámbito del sector público, sector privado, personas físicas y público en general, además de promover una manera precisa y ordenada sobre la difusión y uso de los datos geográficos. 
Entre todas estas normas publicada y vigentes, el Perfil Oficial de Metadatos Geográficos de Costa Rica resulta fundamental para el trabajo con proyecciones cartográficas, pues los metadatos serán los que permitan la trazabilidad de los datos geoespaciales al dar respuesta a dos preguntas básicas en el tema de las transformaciones entre proyecciones cartográficas: ¿con qué se cuenta? y ¿a dónde se puede ir?.

Los metadatos son "datos acerca de los Datos" (Olaya, 2014: 767), y su misión es-"mostrar la información de una manera estructurada sobre esos datos". Es decir, ayudan al usuario a elegir y acceder a los datos de una manera más precisa y pronta, por medio de la búsqueda de catálogos, facilitando la calidad de la información que necesitamos.-Los metadatos son un documento adicional que acompañan a los datos, y que permiten una mejor gestión y una utilización más precisa de ellos-

Los metadatos son descriptores en lo que se indica información básica que permiten: conocer (título, palabras clave, resumen, objetivos, propósito, formato, temática, entre otros), quién y cómo se distribuye, como fue generado el dato (metodología), su (representación espacial, sistema de referencia, calidad) y extensión geográfica.

Según se indica en la norma NTIG_CR04_01.2016, los metadatos se generan por medio de plantillas diseñadas en lenguaje XML, las cuales están personalizadas para Costa Rica, basadas en el Perfil Latinoamericano de Metadatos (LAMP), bajo la norma ISO 19115:2003, estas pueden completarse utilizando el programa GeoNetwork o directamente ser accedidas dentro de la plataforma del SNIT. En total son cuatro plantillas, dos-en formato vectorial y dos en formato ráster, con y sin el Marco Internacional de Referencia Terrestre (ITRF) que se denominan:

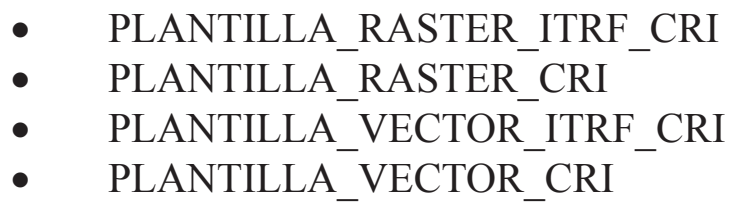

El Perfil Oficial de Metadatos Geográficos de Costa Rica, y por lo tanto las plantillas, contiene una sección denominada Sistema de Referencia, en la que se describe el sistema de referencia espacial y temporal usado en el conjunto de datos (IGN, 2014). En esta sección se debe indicar de forma obligatoria 
Bepsy Cedeño-Montoya, Jorge Moya-Zamora. Impacts resulting from lack of knowledge about the reference system in geospatial information of Costa Rica: utility of metadata

el título o nombre del sistema de la proyección de referencia de los datos, el código EPSG correspondiente, la fecha cuando se genera el elemento y el tipo de fecha (de creación o de levantamiento de los datos), y opcionalmente otros aspectos importantes de mencionar como datum, elipsoide de referencia y la época, que es la fecha vinculada directamente con la realización más actual del ITRF (este último sugerido solo para datos con escalas mayores a 1:1000).

La diferencia entre las plantillas de cada modelo de datos radica en que las que incluyen la palabra ITRF en su nombre solicitan mayor especificidad respecto al sistema de referencia espacial que las otras. Es decir, PLANTILLA_RASTER_ITRF_CRI y PLANTILLA_VECTOR_ITRF_ CRI ofrecen la posibilidad de que en la sección de referencia espacial se complete el apartado "Otros aspectos importantes de mencionar" con la información sobre datum, elipsoide de referencia, ITRF y época; mientras que las otras dos plantillas esta información no se contempla.

Figura 7. Visualización, en ArcCatalogo 10.3, de la sección de referencia de las plantillas de metadatos, según la norma NTIG_ CR04_01.2016. Arriba la plantilla PLANTILLA_VECTOR_CRI y abajo PLANTILLA_VECTOR_ITRF_CRI

Reference System Information

REFERENCE SYSTEM IDENTIFIER

VALUE (obligatorio) Se refiere al código EPSG o código de la proyección de referencia según European Petroleum Survey Group (EPSG).

\footnotetext{
Reference System Information

REFERENCE SYSTEM IDENTIFIER

VALUE (obligatorio) Se refiere al código EPSG o código de la proyección de referencia según European Petroleum Survey Group (EPSG).

AUTHORITY THAT DEFINES THE VALUE

TITLE (obligatorio) Se refiere al nombre del del sistema, o sea la proyección de referencia del dato.

CREATION DATE $2005-10-12$

OTHER CITATION Details (opcional) Este apartado debe de completarse tomando en cuenta los siguientes cuatro aspectos: 1.Datum: Se refiere a la materialización del elipsoide de referencia en un territorio, 2. Elipsoide de referencia: Es nombre del elpsoide de referencia, 3. ITRF: Es un conjunto de puntos con sus coordenadas cartesianas en 3 dimensiones distribuidos en todo el mundo y que sirven como un sistema de referencia ideal y 4. Época: Se refiere a la época en la cual se realiza una medición que tiene vínculo directo con la realización más actual del ITRF.
}

Fuente: Elaboración propia

Esta diferenciación de la sección de referencia espacial de las plantillas es "una personalización del perfil para Costa Rica" (IGN, 2016: 22), creada con la finalidad de ofrecer a los usuarios la posibilidad de completar información que puede ser de suma importancia para cierto tipo de datos y trabajos. 
Esta sección de referencia de los metadatos es importante para el trabajo con proyecciones cartográficas, pues permite saber en qué sistema de proyección se encuentran los datos al momento de la consulta, y con esto se logra responder a una primera pregunta básica ¿con qué se cuenta?, lo que será fundamental para analizar ¿a dónde se puede ir?, y así identificar como proyectar los datos al plano correcto o realizar transformaciones de manera adecuada.

A pesar de que desde el año 2007, el CRTM05 es el sistema de proyección oficial de Costa Rica (Decreto ejecutivo 33797-MJ-MOPT), aún es posible encontrar gran cantidad de información geoespacial en otras proyecciones cartográficas, ante lo que los usuarios se ven en la necesidad de realizar transformaciones buscando la representación en el plano oficial. Lamentablemente para muchos de los datos se desconoce el sistema de proyección en el que fueron originados, por lo que la definición y posterior reproyección (entendida como el cambio de entre coordenadas cartográficas) se realiza de forma poco certera. Los procesos correctos de transformación, dependiendo de la fuente original de los datos, se pueden consultar en Moya y Cedeño (2016).

Usualmente, las proyecciones de datos al plano y posteriores reproyecciones suelen realizarse utilizando programas especializados en el manejo de información geográfica, por lo que los usuarios realizan los procedimientos sin cuestionar los resultados; asumiendo que la precisión de los datos de entrada es igual a la de los datos resultantes. Y aunque los parámetros de cada sistema de proyección hayan sido incluidos correctamente en el software, no necesariamente las transformaciones se realizan adecuadamente, por lo que es importante conocer con claridad y certeza cuál es el plano original en el que se crearon los datos y seguir el camino correcto para su transformación hacia otros sistemas proyectivos.

\section{Conclusiones}

Las diferencias entre sistemas de proyección como CRTM90, CRTM98 rondan en promedio uno 7,5 m (Dörries y Roldán, 2004), producto de la falta de vínculo al ITRF en el caso del datum costarricense CR90. Las diferencias entre coordenadas CRTM98 y CRTM05, haciendo la adecuación respectiva del factor de escala del meridiano central, puede llegar a valores de $50 \mathrm{~cm}$ en algunos casos, dependiendo de la ubicación dentro del territorio nacional. Lo 
Bepsy Cedeño-Montoya, Jorge Moya-Zamora. Impacts resulting from lack of knowledge about the reference system in geospatial information of Costa Rica: utility of metadata

anterior, no permite visualizar un efecto directo en el producto cartográfico de escalas superiores a 1:1000, pero que si suma error a trabajos de escalas mayores realizados con herramientas de alta precisión. Adicionalmente, transformaciones de datum como las que se debe realizar al trasladar datos entre los sistemas de proyección LCRN o LCRS, y CRTM05, pueden generar diferencias hasta de metros (ver cuadro 2 y 3 ), si no son realizadas correctamente.

El trabajo con SIG y los Sistemas Satelitales de Navegación Global (GNSS) se ha extendido a una gran cantidad de usuarios con o sin formación profesional en geociencias. Además, en Costa Rica existe información referida a muchos datum y proyecciones cartográficas, lo que obliga a los usuarios a "reproyectar" los datos de un sistema de coordenadas a otro (Moya y Cedeño, 2016).

El programa ArcGIS, versión 10.3, integra en las herramientas de proyecciones y transformaciones una serie de parámetros que permiten la conversión y transformación de los datos espaciales a otras referencias en caso necesario, pero los resultados de su aplicación dependen del conocimiento que tenga el usuario. Se ha notado que, para una gran mayoría de los usuarios SIG hay confusión conceptual entre algunos términos, por ejemplo: elipsoide, datum, sistema de coordenadas, proyección, reproyección y transformación entre otros, la cual se incrementa con la nomenclatura usada directamente por la herramienta SIG. Una base conceptual general sobre términos relacionados con geodesia y proyecciones cartográficas, se puede tener consultando a Moya y Cedeño (2016a).

Se han tomado como datos de partida coordenadas geodésicas referidas a los dos principales datum de Costa Rica, el convencional Ocotepeque, elipsoide Clarke 1866, y el actual y oficial datum CR05, elipsoide WGS84. Además, se consideraron coordenadas cartográficas basadas en las proyecciones Lambert Costa Rica Norte (LCRN) y CRTM05. Los resultados de este estudio contemplaron los siguientes aspectos:

Como resultado principal del caso A, se comprueban los $265 \mathrm{~m}$ de diferencia promedio entre el datum convencional Ocotepeque y el datum CR98, tal como lo expusieron Dörries y Roldán (2004). Los parámetros de transformación determinados como producto del estudio realizado por estos autores condujeron a un conjunto de parámetros de transformación, que son los mismos que están integrados dentro de las herramientas de georreferenciación del ArcGIS versión 10.3, como se muestra en la figura 8: 
Bepsy Cedeño-Montoya, Jorge Moya-Zamora. Repercusiones producto del desconocimiento del sistema de referencia en la información geoespacial de Costa Rica: utilidad de los metadatos
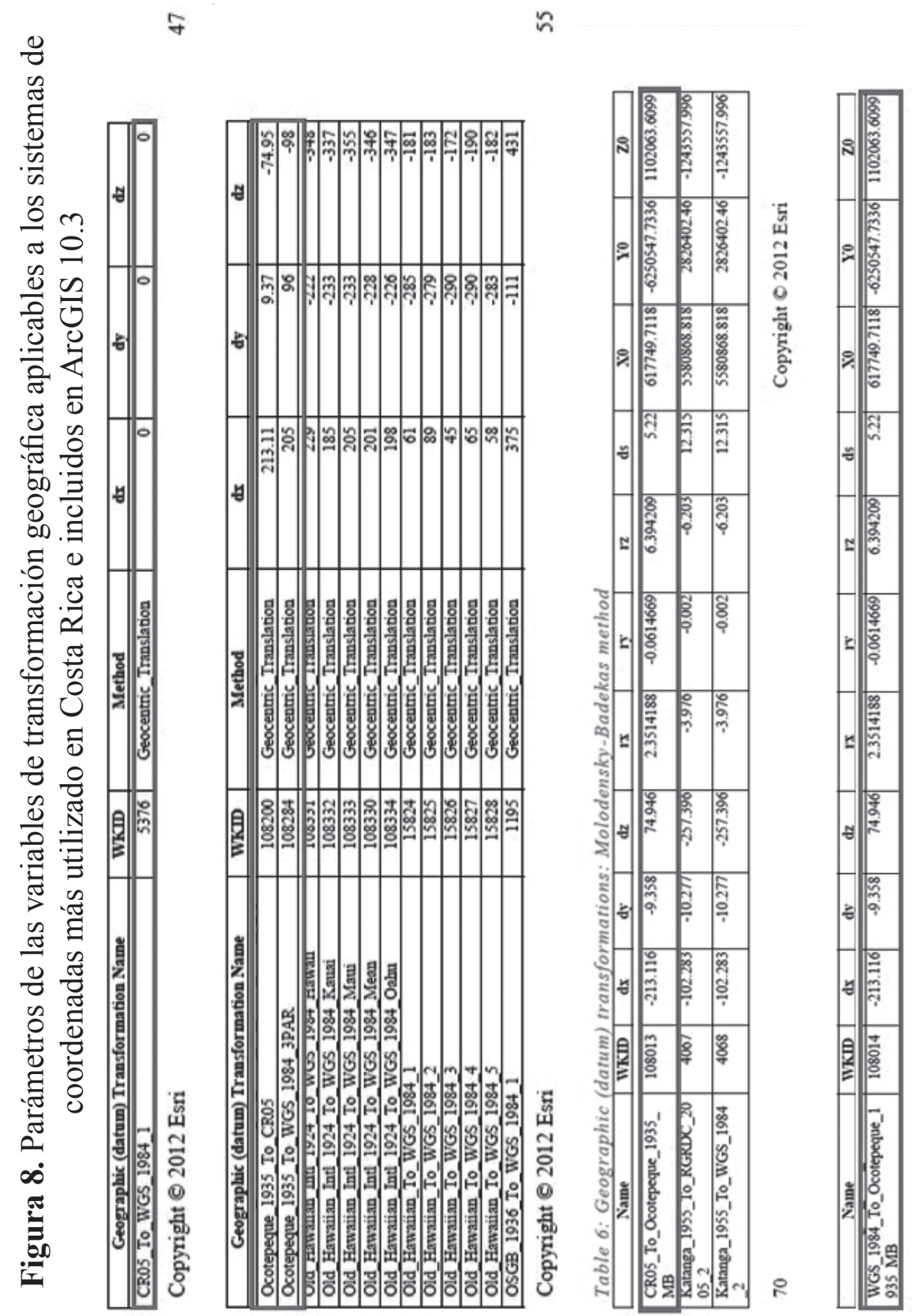

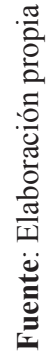


Bepsy Cedeño-Montoya, Jorge Moya-Zamora. Impacts resulting from lack of knowledge about the reference system in geospatial information of Costa Rica: utility of metadata

Se demuestra, como al realizar el ejercicio de considerar como elipsoide de partida WGS84 en lugar de Clarke 1866 y su respectivo datum, se encuentran diferencias como las citadas anteriormente (ver cuadro 2 y cuadro 3). Estas diferencias a nivel nacional se presentan en la figura 3, donde se tiene un desplazamiento en sentido sureste. Por otro lado, una buena lectura de la información de partida en el caso A, produce resultados nulos al calcular coordenadas cartográficas LCRN a partir de coordenadas geodésicas, datum Ocotepeque, elipsoide Clarke 1866 (ver cuadro 4).

El caso B tomó como insumo coordenadas geodésicas referidas al datum nacional CR05, elipsoide WGS84 y se calcularon coordenadas cartográficas CRTM05. Evidentemente, al comparar con los valores oficiales de coordenadas cartográficas se obtuvieron diferencias nulas.

Los cálculos realizados bajo el apartado $\mathrm{C}$ demostraron que existen diferencias, según se use la variable de transformación CR05_to_Ocotepeque_1935_MB en un sentido o en otro. Del cuadro 5 se evidencia un rango de diferencia aproximada de 4,1 $\mathrm{m}$ en la coordenada norte y de 5,8 $\mathrm{m}$ en la coordenada este usando la variable en sentido directo. La aplicación de la variable en sentido inverso refleja rangos de diferencias en las coordenadas de 5,7 $\mathrm{m}$ en el norte y de 6,9 $\mathrm{m}$ en la coordenada este. Se debe recalcar que, aunque el promedio de las diferencias sea de $70 \mathrm{~cm} \pm 14 \mathrm{~cm}$ y de 82 $\mathrm{cm} \pm 15 \mathrm{~cm}$ para el uso de las variables en sentido directo e inverso, el rango de diferencias dependerá de la zona de trabajo.

Los resultados del apartado D demostraron que existen diferencias en el proceso al usar la variable de transformación Ocotepeque_1935_to_ CR05 de forma directa e inversa. Los resultados llaman la atención en dos aspectos principales: por un lado, se puede decir que las dos variables de transformación en el SIG brindan resultados prácticamente iguales para los valores extremos, sin embargo, debe considerarse que los resultados dependerán de la zona de trabajo. Por ejemplo, los resultados producto de la aplicación directa de esta variable produjeron diferencia con un rango de 21,0 m para la coordenada norte y para la coordenada este de 55,5 m. Usando la misma variable en sentido inverso, el rango de diferencias fue de 20,0 m en la coordenada norte y de 54,5 m en la coordenada este En la figura 9 se presenta la comparación de los vectores diferencia para las zonas de puntos correspondientes a LCRN (parte superior) y LCRS (parte inferior). El color rojo de los vectores corresponde a los resultados al aplicar 
la variable, mientras que el color azul representa la aplicación al aplicar la variable de manera inversa. En segundo lugar, la comparación de los resultados dados por el SIG con las coordenadas oficiales referidas a CRTM98, presentaron promedio de diferencias prácticamente iguales de $43 \mathrm{~cm} \pm 4$ $\mathrm{cm}$ para las coordenadas norte y de $49 \mathrm{~cm} \pm 5 \mathrm{~cm}$. Estos datos reflejan un vector diferencia de unos $65 \mathrm{~cm} \pm 4,4 \mathrm{~cm}$ producto de esta comparación.

La creación de los metadatos es indispensable y debe de ir ligada a la información geoespacial, por lo tanto, todos los generadores de información deben preocuparse por completar adecuadamente las diferentes secciones que conforman las plantillas de metadatos. En el caso de la norma NTIG CR04_01_2016 Perfil oficial de metadatos Geográficos de Costa Rica, las plantillas solicitan que se indique el Título y Código EPSG, requerimientos mínimos necesarios para un adecuado tratamiento de los datos. No obstante, las plantillas también contienen campos para indicar otros aspectos igualmente importantes como el datum, elipsoide de referencia y la época, elementos que tendrán mayor relevancia dependiendo de la escala final del producto.

Los objetivos de cada uno de los proyectos son los que condicionan el tratamiento que se le da a la información, sin embargo, aunque para muchos productos cartográficos las diferencias encontradas pueden suprimirse, no es adecuado omitir el proceso correcto de vinculación de la información de partida. En muchos casos, la escala cartográfica de los productos no permite distinguir visualmente las posibles diferencias que existan por un inadecuado manejo de la información, pero lo anterior no exime a los usuarios de hacer un proceso correcto tanto desde el punto de vista matemático como del cartográfico.

Los programas SIG son herramientas sumamente poderosas y con un altísimo potencial de trabajo. Los resultados han demostrado una vez más que los sistemas informáticos responderán dependiendo de la información de partida, la cual es responsabilidad de los generadores de datos y posteriormente de los usuarios de los sistemas, quienes se encargan de generar los diferentes productos.

\section{Recomendaciones}

Es necesario que el usuario conozca y maneje los conceptos geodésicos y cartográficos básicos para lograr entender el funcionamiento de los Sistemas de Información Geográfica, y, por consiguiente, hacer una 
Bepsy Cedeño-Montoya, Jorge Moya-Zamora. Impacts resulting from lack of knowledge about the reference system in geospatial information of Costa Rica: utility of metadata

adecuada y correcta configuración de la herramienta. Por ejemplo, no se debe asumir que los valores de coordenadas geodésicas están necesariamente referidos al elipsoide WGS84, ni que en caso de contar con coordenadas cartográficas, éstas sean basadas en cartografía Lambert, ya que en Costa Rica se han manejado dos sistemas de esta proyección. De igual forma, valores de coordenadas cartográficas asumidos en CRTM05 pueden ser también valores dados en las proyecciones CRTM98 o CRTM90.

El programa contiene herramientas informáticas poderosas desde el punto de vista del manejo de grandes volúmenes de datos, sin embargo, es altamente recomendable que el usuario pueda corroborar los resultados que se dan como producto final.

Se recomienda ampliamente a los usuarios que tengan dudas respecto a la configuración del SIG hacer la revisión respectiva, o paralelamente hacer la conversión de coordenadas por medio de una programación dentro del propio SIG, o en caso contrario, hacer fuera del ambiente del SIG las conversiones y transformaciones necesarias de manera que pueda asegurarse que los datos geoespaciales están adecuadamente georreferenciados.

En general, ante la ausencia de los metadatos en gran parte de la información actual que existe en el país, y particularmente, cuando la fuente de la información desconoce o tiene ciertas dudas sobre el sistema de referencia de los datos, es imperativo conocer la fuente de la información geoespacial, ya que de ella dependerán los procesos posteriores de conversión y transformación de coordenadas adecuados para la generación de productos cartográficos de calidad.

Desde inicios del año 2016, el IGN ha puesto a disposición de los usuarios las plantillas para la creación de los metadatos, las cuales deben ser de uso diario para los generadores de datos geoespaciales. Contar con los elementos mínimos del sistema de referencia (título y código EPSG) es fundamental para los procesos de conversión y transformación de los datos espaciales. En trabajos de alta precisión es recomendable utilizar las plantillas ráster o vector con ITRF para ofrecer la información adicional y útil referente al datum, al elipsoide de referencia y época. 


\section{Referencias}

Decreto ejecutivo 33797-MJ-MOPT. Ministerio de Justica y Gracia. Costa Rica. Diario Oficial La Gaceta. San José, Costa Rica. 12 de julio de 2013

Dörries, E. y Roldán, J. (2004). El Datum Geodésico de Ocotepeque y el Datum Satelitario del Sistema WGS84. Revista UNICIENCIA, 21(1 y 2), 117-125.

Environmental Systems Research Institute (ESRI). (2012). ArcGIS 10.1 Geographic and Vertical Transformation Tables. California, EEUU. Disponible en la documentación de instalación del programa ArcGIS, en la ruta: C: $\backslash$ Program Files (x86)\ArcGIS \Desktop10.3 \Documentation $\backslash$ geographic_transformations.pdf

Instituto Geográfico Nacional. (2001). División Territorial Administrativa de la República de Costa Rica. Instituto Geográfico Nacional. Departamento de División Territorial y Nomenclatura, (4 ed). San José. ISBN 9977-58-215-7

Instituto Geográfico Nacional. (2016). NTIG_CR04_01.2016: Perfil Oficial de Metadatos Geográficos de Costa Rica. San José: Costa Rica. Instituto Geográfico Nacional, Registro Nacional. Recuperado de http://www.snitcr.go.cr/index.php?option=com_content\&view=arti cle\&id=59:normativa

Moya, J. y Cedeño, B. (2016a). Conceptos Básicos en Geodesia como Insumo para un Tratamiento Adecuado de la Información Geoespacial. Revista Geográfica de América Central, 58(1), 71-100. Doi: http:// dx.doi.org/10.15359/rgac.58-1.3

Moya, J. y Cedeño, B. (2016b). Los diferentes datum y proyecciones cartográficas de Costa Rica: generalidades y relaciones. Revista Geográfica de América Central, 59(3). Doi: http://dx.doi.org/10.15359/ rgac.3-59.2

Olaya, V. (2011). Sistemas de Información Geográfica. Versión revisada el 16 de octubre de 2014. Recuperado de http://volaya.github.io/ libro-sig/ 